This is an electronic reprint of the original article. This reprint may differ from the original in pagination and typographic detail.

Author(s): Juutinen, Petri

Title: Decay estimates in the supremum norm for the solutions to a nonlinear evolution equation

Year: $\quad 2014$

Version:

Please cite the original version:

Juutinen, P. (2014). Decay estimates in the supremum norm for the solutions to a nonlinear evolution equation. Proceedings of the Royal Society of Edinburgh, Section: A Mathematics, 144(3), 557-566. https://doi.org/10.1017/S0308210512001163

All material supplied via JYX is protected by copyright and other intellectual property rights, and duplication or sale of all or part of any of the repository collections is not permitted, except that material may be duplicated by you for your research use or educational purposes in electronic or print form. You must obtain permission for any other use. Electronic or print copies may not be offered, whether for sale or otherwise to anyone who is not an authorised user. 


\title{
DECAY ESTIMATES IN THE SUPREMUM NORM FOR THE SOLUTIONS TO A NONLINEAR EVOLUTION EQUATION
}

\author{
PETRI JUUTINEN
}

\begin{abstract}
We study the asymptotic behavior, as $t \rightarrow \infty$, of the solutions to the nonlinear evolution equation

$$
u_{t}=\Delta_{p}^{N} u
$$

where $\Delta_{p}^{N} u=\Delta u+(p-2)\left(D^{2} u \frac{D u}{|D u|}\right) \cdot \frac{D u}{|D u|}$ is the normalized $p$-Laplace equation and $p \geq 2$. We show that if $u(x, t)$ is a viscosity solution to the above equation in a cylinder $\Omega \times(0, \infty)$ with time-independent lateral boundary values, then it converges to the unique stationary solution $h$ as $t \rightarrow \infty$. Moreover, we provide an estimate for the decay rate of $\max _{x \in \Omega}|u(x, t)-h(x)|$.
\end{abstract}

\section{INTRODUCTION}

A systematic study of the nonlinear evolution equation

$$
u_{t}=\Delta_{p}^{N} u, \quad 1<p<\infty
$$

where

$$
\begin{aligned}
\Delta_{p}^{N} u & =|D u|^{2-p} \operatorname{div}\left(|D u|^{p-2} D u\right) \\
& =\Delta u+(p-2)\left(D^{2} u \frac{D u}{|D u|}\right) \cdot \frac{D u}{|D u|}
\end{aligned}
$$

is the normalized $p$-Laplace operator, has been recently initiated by Does [6] and Banerjee and Garofalo [2]. Equation (1.1) arises from image processing [6] and random turn games [12], and it can also be viewed as a generalization of either the motion of level sets by mean curvature (the case $p=1$ ) or the classical heat equation. Compared to the more familiar $p$-parabolic equation

$$
u_{t}=\operatorname{div}\left(|D u|^{p-2} D u\right),
$$

(1.1) has the advantage that it is 1-homogeneous, but also the severe disadvantage that it is not of divergence form.

The purpose of this note is to complement a result obtained by Banerjee and Garofalo in [2]. They proved, among other things, that if $1<p \leq 2$ and $u=u(x, t)$ is the unique viscosity solution to the Cauchy-Dirichlet problem

$$
\begin{cases}u_{t}=\Delta_{p}^{N} u & \text { in } \Omega \times(0, \infty), \\ u(x, 0)=\varphi(x) & \text { for } x \in \Omega, \\ u(x, t)=\varphi(x) & \text { for } x \in \partial \Omega, t>0\end{cases}
$$

Date: October 19, 2015.

2000 Mathematics Subject Classification. 35K55, 35K65, 35B40.

Key words and phrases. asymptotic behavior, $p$-Laplace equation. 
with $\Omega \subset \mathbb{R}^{n}$ open and bounded, then $u(x, t) \rightarrow h(x)$, where $h$ is the unique $p$-harmonic function in $\Omega$ having boundary values $\varphi$ on $\partial \Omega$. Observe that it is assumed that the values of $u$ on the lateral boundary of $\Omega \times(0, \infty)$ are independent of $t$.

However, the case $p>2$ was left open. We show in this paper that the same result holds also in the case $p>2$ and, moreover, provide an estimate for the decay rate of $\max _{x \in \Omega}|u(x, t)-h(x)|$. Our proof relies on maximum principle techniques and it is completely different from the approach used in [2] in which monotonicity of the energy played a key role.

In order to describe our method, let us suppose that $u$ is a smooth solution to $(1.2)$, and let $h$ be the unique $p$-harmonic function such that $h=\varphi$ on $\partial \Omega$, that is,

$$
\begin{cases}-\operatorname{div}\left(|D h|^{p-2} D h\right)=0 & \text { in } \Omega, \\ h(x)=\varphi(x) & \text { for } x \in \partial \Omega .\end{cases}
$$

If we can find functions $v^{ \pm}$such that $v_{t}^{+}-\Delta_{p}^{N} v^{+} \geq 0, v_{t}^{-}-\Delta_{p}^{N} v^{-} \leq 0$, and $v^{-} \leq u \leq v^{+}$on the parabolic boundary of $\Omega \times(0, \infty)$, then $v^{-} \leq u \leq v^{+}$ by the comparison principle and we have

$\|u(x, t)-h(x)\|_{L^{\infty}(\Omega)} \leq \max \left\{\left\|v^{+}(x, t)-h(x)\right\|_{L^{\infty}(\Omega)},\left\|v^{-}(x, t)-h(x)\right\|_{L^{\infty}(\Omega)}\right\}$.

Thus, finding "good" barriers $v^{+}$and $v^{-}$that converge to $h$ would give an estimate on the decay of $|u(x, t)-h(x)|$ as $t \rightarrow \infty$.

Let us now focus on the upper barrier $v^{+}$and call it simply $v$ for the rest of this discussion. Since $v$ should converge to $h$ as $t \rightarrow \infty$, we make an ansatz that $v$ is of the form

$$
v(x, t)=h(x)+f(t, h(x))
$$

for some function $f=f(t, h)$ which is decreasing in $t$ and satisfies $f(t, h) \rightarrow 0$ as $t \rightarrow \infty$. Since $D v=\left(1+f_{h}\right) D h$ and $D^{2} v=\left(1+f_{h}\right) D^{2} h+f_{h h} D h \otimes D h$, we have

$$
\begin{aligned}
v_{t}-\Delta_{p}^{N} v & =f_{t}-\left(1+f_{h}\right) \Delta_{p}^{N} h-(p-1) f_{h h}|D h|^{2} \\
& =f_{t}-(p-1) f_{h h}|D h|^{2},
\end{aligned}
$$

where the fact that $-\Delta_{p}^{N} h=0$ was used. The term $f_{t}$ is negative because $f$ is decreasing, and so, to have that $v_{t}-\Delta_{p}^{N} v \geq 0$, we need the term $f_{h h}|D h|^{2}$ to be negative and even bounded away from zero. This, however, would require that $D h$ does not vanish, which is a far too restrictive assumption.

Since we are not aware of a method that would enable us to approximate $h$ by solutions with non-vanishing gradient (unless $p=\infty$, see [1]), we instead replace $h$ by a solution $h_{\varepsilon}$ of an approximating equation $-\Delta_{p}^{N} h_{\varepsilon}=\varepsilon$ and set $v(x, t)=h_{\varepsilon}(x)+f\left(t, h_{\varepsilon}(x)\right)$. Then the above computation yields

$$
v_{t}-\Delta_{p}^{N} v \geq f_{t}+\left(1+f_{h}\right) \varepsilon,
$$

which can be made positive by choosing $f$ appropriately (depending on $\varepsilon$ ). Observe that now $v(x, t) \rightarrow h_{\varepsilon}(x)$ as $t \rightarrow \infty$, and thus, to make the argument work, we need to estimate $\left\|h-h_{\varepsilon}\right\|_{\infty, \Omega}$ and balance this with the dependence of $f$ on $\varepsilon$. 
It seems to us that estimating $\left\|h-h_{\varepsilon}\right\|_{\infty, \Omega}$ would require sophisticated game theoretic arguments (precise estimates on the expected number of rounds in the game etc.), whereas the difference of $h$ and the solution $\tilde{h}_{\varepsilon}$ of

$$
-\left|D \tilde{h}_{\varepsilon}\right|^{p-2} \Delta_{p}^{N} \tilde{h}_{\varepsilon}=\varepsilon
$$

is much easier to estimate due to the structural properties of the standard $p$-Laplacian $-|D w|^{p-2} \Delta_{p}^{N} w=\operatorname{div}\left(|D w|^{p-2} D w\right)$. Hence we in fact construct our barrier $v(x, t)$ using $\tilde{h}_{\varepsilon}$ instead of $h_{\varepsilon}$, which, however, brings along the need to bound $\|D h\|_{\infty, \Omega}^{2-p}$ from below. But this can be done as long as $p>2$ and $\Omega$ and $\varphi$ are smooth.

The precise statement about the decay as well as its proof are given in Section 3 below, apart from the estimate for $\left\|h-\tilde{h}_{\varepsilon}\right\|_{\infty, \Omega}$ that is proved in Section 4 . The special case where $\varphi(x)=0$ on $\partial \Omega$ is also briefly discussed in Section 3.

\section{Definitions}

There are several equivalent ways to define viscosity solutions for (1.1). We have chosen to use the definition that involves semicontinuous envelopes of the function $F(\xi, M)=\operatorname{trace}(M)+(p-2)\left(M \frac{\xi}{|\xi|}\right) \cdot \frac{\xi}{|\xi|}$. For simplicity, and since our main result only deals with the case $p>2$, we have stated the definition only for these values of $p$. For $1<p<2$, the envelopes take a slightly different form, see e.g. [2]. We refer the reader to [7], [5] and [8] for the justification of the definition and the basic properties such as stability etc., and to [2] for a detailed discussion on an alternative way to define the solutions.

For a symmetric $n \times n$-matrix $A$, we denote its largest and smallest eigenvalue by $\Lambda(A)$ and $\lambda(A)$, respectively. That is,

$$
\Lambda(A)=\max _{|\eta|=1}(A \eta) \cdot \eta
$$

and

$$
\lambda(A)=\min _{|\eta|=1}(A \eta) \cdot \eta
$$

Definition 2.1. Let $D \subset \mathbb{R}^{n+1}$ be an open set. An upper semicontinuous function $u: D \rightarrow \mathbb{R}$ is a viscosity subsolution of (1.1) in $D$ if, whenever $(\hat{x}, \hat{t}) \in D$ and $\varphi \in C^{2}(D)$ are such that

(1) $u(\hat{x}, \hat{t})=\psi(\hat{x}, \hat{t})$

(2) $u(x, t)<\psi(x, t)$ for all $(x, t) \in D,(x, t) \neq(\hat{x}, \hat{t})$

then

$$
\left\{\begin{array}{lc}
\psi_{t}(\hat{x}, \hat{t}) \leq \Delta_{p}^{N} \psi(\hat{x}, \hat{t}) & \text { if } D \psi(\hat{x}, \hat{t}) \neq 0 \\
\psi_{t}(\hat{x}, \hat{t}) \leq \Delta \psi(\hat{x}, \hat{t})+(p-2) \Lambda\left(D^{2} \psi(\hat{x}, \hat{t})\right) & \text { if } D \psi(\hat{x}, \hat{t})=0 .
\end{array}\right.
$$

A lower semicontinuous function $u: D \rightarrow \mathbb{R}$ is a viscosity supersolution of (1.1) in $D$ if $-u$ is a viscosity subsolution, that is, whenever $(\hat{x}, \hat{t}) \in D$ and $\psi \in C^{2}(D)$ are such that

(1) $u(\hat{x}, \hat{t})=\psi(\hat{x}, \hat{t})$

(2) $u(x, t)>\psi(x, t)$ for all $(x, t) \in D,(x, t) \neq(\hat{x}, \hat{t})$ 
then

$$
\left\{\begin{array}{lc}
\psi_{t}(\hat{x}, \hat{t}) \geq \Delta_{p}^{N} \psi(\hat{x}, \hat{t}) & \text { if } D \psi(\hat{x}, \hat{t}) \neq 0 \\
\psi_{t}(\hat{x}, \hat{t}) \geq \Delta \psi(\hat{x}, \hat{t})+(p-2) \lambda\left(D^{2} \psi(\hat{x}, \hat{t})\right) & \text { if } D \psi(\hat{x}, \hat{t})=0
\end{array}\right.
$$

Finally, a continuous function $u: D \rightarrow \mathbb{R}$ is a viscosity solution of $(1.1)$ in $D$ if it is both a viscosity subsolution and a viscosity supersolution.

\section{MAIN RESUlt AND ITS PROOF}

Here is our main result:

Theorem 3.1. Let $\Omega$ be a smooth, bounded domain in $\mathbb{R}^{n}$. Let $\varphi \in C^{2}(\bar{\Omega})$ and suppose that $h \in C(\bar{\Omega})$ is the unique p-harmonic function in $\Omega$ satisfying $h=\varphi$ on $\partial \Omega$. Let further $u \in C(\bar{\Omega} \times[0, \infty)$ be the unique solution to

$$
\begin{cases}u_{t}=\Delta_{p}^{N} u & \text { in } \Omega \times(0, \infty), \\ u(x, 0)=\varphi(x) & \text { for } x \in \Omega, \\ u(x, t)=\varphi(x) & \text { for } x \in \partial \Omega, t>0 .\end{cases}
$$

Then, if $p>2$, for each $q>1$ there exists $C=C(p, n, q, \Omega, \varphi)>0$ such that

$$
\sup _{x \in \Omega}|u(x, t)-h(x)| \leq C(1+t)^{-\frac{1}{q(p-1)}} \quad \text { for } t>0 .
$$

Proof. For $\varepsilon>0$, let $h_{\varepsilon}^{ \pm}$be the unique solutions to

$$
\begin{cases}-\operatorname{div}\left(\left|D h_{\varepsilon}^{ \pm}\right|^{p-2} D h_{\varepsilon}^{ \pm}\right)= \pm \varepsilon & \text { in } \Omega \\ h_{\varepsilon}^{ \pm}=\varphi & \text { on } \partial \Omega .\end{cases}
$$

Then, by comparison principle, $h_{\varepsilon}^{-} \leq h \leq h_{\varepsilon}^{+}$in $\Omega$, and Theorem 4.1 below implies that there exists a constant $C=C(p, n, \Omega)$ such that

$$
\sup _{\Omega}\left|h-h_{\varepsilon}^{ \pm}\right| \leq C \varepsilon^{1 /(p-1)} .
$$

By adding a constant if necessary, we may assume that $h(x) \geq 1$ in $\Omega$ and $u(x, t) \geq 1$ in $\Omega \times(0, \infty)$.

For $\mu>0$, let us denote

$$
v(x, t)=S(t) h_{\varepsilon}^{+}(x), \quad \text { where } S(t)=1+M e^{-\mu t} \text { and } M=\|\varphi\|_{\infty, \Omega} .
$$

Then, as $h_{\varepsilon}^{+} \geq 1$,

$$
v(x, 0) \geq(1+M) h_{\varepsilon}^{+}(x) \geq M \geq \varphi(x) \quad \text { for all } x \in \Omega
$$

and

$$
v(x, t) \geq h_{\varepsilon}^{+}(x)=\varphi(x) \quad \text { on } \partial \Omega \times(0, \infty) .
$$

Thus $v \geq u$ on the parabolic boundary of $\Omega \times(0, \infty)$. Moreover,

$$
\begin{aligned}
v_{t}-\Delta_{p}^{N} v & =S^{\prime}(t) h_{\varepsilon}^{+}(x)-S(t) \Delta_{p}^{N} h_{\varepsilon}^{+}(x) \\
& =-\mu M e^{-\mu t} h_{\varepsilon}^{+}(x)+\varepsilon\left(1+M e^{-\mu t}\right)\left|D h_{\varepsilon}^{+}(x)\right|^{2-p} \\
& \geq-\mu M e^{-\mu t}\left\|h_{\varepsilon}^{+}\right\|_{\infty, \Omega}+\varepsilon M e^{-\mu t}\left\|D h_{\varepsilon}^{+}\right\|_{\infty, \Omega}^{2-p},
\end{aligned}
$$


where we used the fact that $2-p \leq 0$. Hence $v_{t}-\Delta_{p}^{N} v \geq 0$ in $\Omega \times(0, \infty)$ provided that

$$
0<\mu \leq L \varepsilon \quad \text { with } L=\frac{\left\|D h_{\varepsilon}^{+}\right\|_{\infty, \Omega}^{2-p}}{\left\|h_{\varepsilon}^{+}\right\|_{\infty, \Omega}} ;
$$

recall that we have assumed that $\left\|h_{\varepsilon}^{+}\right\|_{\infty, \Omega} \geq\|h\|_{\infty, \Omega} \geq 1$.

Since $v_{t}-\Delta_{p}^{N} v \geq 0$ in $\Omega \times(0, \infty)$ and $v \geq h$ on the parabolic boundary of $\Omega \times(0, \infty)$, it follows from the comparison principle and (3.2) that

$$
\begin{aligned}
u(x, t) & \leq v(x, t) \leq h_{\varepsilon}^{+}(x)+M\left\|h_{\varepsilon}^{+}\right\|_{\infty, \Omega} e^{-\mu t} \\
& \leq h(x)+C \varepsilon^{\frac{1}{p-1}}+M\left\|h_{\varepsilon}^{+}\right\|_{\infty, \Omega} e^{-\mu t}
\end{aligned}
$$

for all $t>0$ and $\varepsilon>0$. For a fixed $t>0$, by choosing $\varepsilon=t^{-\frac{1}{q}}$ and recalling that we may take $\mu=L \varepsilon$, this yields

$$
u(x, t) \leq h(x)+C\left(t^{-\frac{1}{q(p-1)}}+e^{-t^{1-\frac{1}{q}}}\right) .
$$

If $q>1$, then $e^{-t^{1-\frac{1}{q}}}$ decays faster than $t^{-\frac{1}{q(p-1)}}$, and thus we obtain

$$
u(x, t) \leq h(x)+C(1+t)^{-\frac{1}{q(p-1)}}
$$

for some constant $C=C(p, n, q, \Omega, \varphi)>0$.

A symmetric argument, involving $h_{\varepsilon}^{-}$instead of $h_{\varepsilon}^{+}$, shows that

$$
u(x, t) \geq h(x)-C(1+t)^{-\frac{1}{q(p-1)}},
$$

and thus we have proved Theorem 3.1.

Remark 3.2. Since $\Omega$ is smooth and $\varphi \in C^{2}(\bar{\Omega})$, the functions $h, h_{\varepsilon}^{ \pm} \in$ $C^{1, \alpha}(\bar{\Omega})$ for some $\alpha>0$, see [10]. In particular, $\left\|D h_{\varepsilon}^{ \pm}\right\|_{\infty, \Omega}$ can be bounded in terms of $\|D \varphi\|_{\infty, \partial \Omega}$ and $\left\|D h_{\varepsilon}^{ \pm}\right\|_{p, \Omega}$. Moreover, from the fact that $h_{\varepsilon}^{ \pm}$ minimizes

$$
\int_{\Omega} \frac{1}{p}|D w|^{p} \pm \varepsilon w d x
$$

in $W_{\varphi}^{1, p}(\Omega)$, it follows that for $0<\varepsilon \leq 1$ the quantity $\left\|D h_{\varepsilon}^{ \pm}\right\|_{p, \Omega}$ can be bounded in terms of $\|D \varphi\|_{p, \Omega}$.

Remark 3.3. Since the functions $h_{\varepsilon}^{ \pm}$are, in general, not twice differentiable, the calculation leading to $v_{t}-\Delta_{p}^{N} v \geq 0$ in the proof above is not fully rigorous. However, it is straightforward to verify that $v_{t}-\Delta_{p}^{N} v \geq 0$ does indeed hold in the viscosity sense. To this end, a useful observation is that if $\psi \in C^{2}(\Omega)$ is such that $h_{\varepsilon}^{+}-\psi$ has a local minimum at some $\hat{x} \in \Omega$, then $D \psi(\hat{x}) \neq 0$, because otherwise we would contradict $-\Delta_{p} \psi(\hat{x}) \geq \varepsilon$.

3.1. Vanishing boundary values. In the special case where $u(x, t)=0$ on the lateral boundary of $\Omega \times(0, \infty)$ we have that $u \rightarrow 0$ exponentially fast as $t \rightarrow \infty$. To see this, we recall from [4], see also [3], that there is $\lambda=\lambda(\Omega)>0$ such that the eigenvalue problem

$$
\begin{cases}-\Delta_{p}^{N} w=\lambda w & \text { in } \Omega \\ w=0 & \text { on } \partial \Omega .\end{cases}
$$


has a positive viscosity solution $w_{\Omega} \in C(\bar{\Omega})$; here $\lambda$ is given by

$$
\lambda=\sup \left\{\mu \in \mathbb{R}: \Delta_{p}^{N}+\mu I \text { satisfies the maximum principle }\right\} .
$$

Let now $B_{R}$ be a ball that compactly contains $\Omega$ and let

$$
v(x, t)=C e^{-\mu t} w_{B_{R}}(x), \quad \mu=\lambda\left(B_{R}\right) .
$$

Then $v$ is a solution to (3.1), and $v \geq u$ on the parabolic boundary if

$$
C \geq \frac{\sup _{\Omega} \varphi}{\inf _{\Omega} w_{B_{R}}} .
$$

Thus, by comparison principle, we have

$$
u(x, t) \leq C e^{-\mu t} \quad \text { for }(x, t) \in \Omega \times(0, \infty) .
$$

A lower bound is derived analogously.

By arguing as in [1], one can verify that $u(x, t) \rightarrow h(x)$ exponentially fast also if $|D h(x)| \geq c>0$ in $\Omega$ for some $c>0$. We conjecture that the exponential decay is true in general, that is, the decay estimate in Theorem 3.1 can be improved to $\|u(x, t)-h(x)\|_{L^{\infty}(\Omega)} \leq C e^{-\lambda t}$ for some $\lambda, C>0$.

\section{4. $\varepsilon$-APPROXIMATION OF $p$-HARMONIC FUNCTIONS}

In this section, we prove a sharp estimate for the difference $\left|h-h_{\varepsilon}^{ \pm}\right|$. For the $L^{1}$ norm such an estimate follows quite easily, but to obtain it for the $L^{\infty}$ norm some extra work is needed. The proof below is based on arguments found in [9] and [11].

Theorem 4.1. Let $\varepsilon>0, p>2$, and $h \in W^{1, p}(\Omega)$ be p-harmonic. If $-\Delta_{p} h_{\varepsilon}^{ \pm}= \pm \varepsilon$ in $\Omega$ and $h_{\varepsilon}^{ \pm}-h \in W_{0}^{1, p}(\Omega)$, then there is $C(p, n, \Omega)>0$ such that

$$
\sup _{\Omega}\left|h-h_{\varepsilon}^{ \pm}\right| \leq C \varepsilon^{1 /(p-1)} .
$$

Proof. Due to symmetry, it is enough to estimate the difference $\sup _{\Omega}\left|h-h_{\varepsilon}^{+}\right|$.

Let us begin with some preliminary estimates that in fact suffice in the case $p>n$. First, we verify that $\sup _{\Omega}\left|h-h_{\varepsilon}^{+}\right|$is finite when $\varphi \in L^{\infty}(\Omega)$. To this end, let $R>0$ be such that $\Omega \subset B_{R}(0)$ and let

$$
w(x)=\frac{p-1}{p} \varepsilon^{1 /(p-1)}\left(R^{p /(p-1)}-|x|^{p /(p-1)}\right), \quad 0 \leq|x| \leq R .
$$

Then $-\Delta_{p} w=\varepsilon$ in $B_{R}(0)$ and

$$
\inf _{\partial \Omega} \varphi \leq h \leq h_{\varepsilon}^{+} \leq \sup _{\partial \Omega} \varphi+\frac{p-1}{p} \varepsilon^{1 /(p-1)} R^{p /(p-1)}
$$

by the comparison principle. Thus

$$
\left\|h-h_{\varepsilon}^{+}\right\|_{\infty, \Omega} \leq 2\|\varphi\|_{\infty, \partial \Omega}+C \varepsilon^{1 /(p-1)}
$$

with $C=C(p, \Omega)$.

Next we use $h_{\varepsilon}^{+}-h \in W_{0}^{1, p}(\Omega)$ as a test-function in the weak formulations of $-\Delta_{p} h_{\varepsilon}^{+}=\varepsilon$ and $-\Delta_{p} h=0$ and subtract the resulting equations; this yields

$$
\int_{\Omega}\left(\left|D h_{\varepsilon}^{+}\right|^{p-2} D h_{\varepsilon}^{+}-|D h|^{p-2} D h\right) \cdot D\left(h_{\varepsilon}^{+}-h\right) d x=\varepsilon \int_{\Omega}\left(h_{\varepsilon}^{+}-h\right) d x .
$$


Hence, in view of monotonicity,

$$
\int_{\Omega}\left|D\left(h_{\varepsilon}^{+}-h\right)\right|^{p} d x \leq C \varepsilon \int_{\Omega}\left|h_{\varepsilon}^{+}-h\right| d x .
$$

Combining this with the Sobolev embedding gives

$$
\left\|h_{\varepsilon}^{+}-h\right\|_{1, \Omega} \leq C\left\|D\left(h_{\varepsilon}^{+}-h\right)\right\|_{p, \Omega} \leq C \varepsilon^{1 / p}\left\|h_{\varepsilon}^{+}-h\right\|_{1, \Omega}^{1 / p},
$$

that is,

$$
\left\|h_{\varepsilon}^{+}-h\right\|_{1, \Omega} \leq C \varepsilon^{1 /(p-1)} .
$$

On the other hand, if $p>n$, then (4.3) and Sobolev inequality directly imply

$$
\left\|h_{\varepsilon}^{+}-h\right\|_{\infty, \Omega} \leq C\left\|D\left(h_{\varepsilon}^{+}-h\right)\right\|_{p, \Omega} \leq C \varepsilon^{1 / p}\left\|h_{\varepsilon}^{+}-h\right\|_{\infty, \Omega}^{1 / p},
$$

which is (4.1) for $p>n$.

Thus we are left with the case $1<p \leq n$. For $k>0$, let

$$
A_{k}:=\left\{x \in \Omega: h_{\varepsilon}^{+}-h \geq k\right\}
$$

and $\varphi_{k}=\max \left\{h_{\varepsilon}^{+}-h-k, 0\right\} \in W_{0}^{1, p}\left(A_{k}\right)$. By using $\varphi_{k}$ as a test-function in the weak formulations of $-\Delta_{p} h_{\varepsilon}^{+}=\varepsilon$ and $-\Delta_{p} h=0$ and subtracting the resulting equations we obtain

$$
\int_{A_{k}}\left(\left|D h_{\varepsilon}^{+}\right|^{p-2} D h_{\varepsilon}^{+}-|D h|^{p-2} D h\right) \cdot D\left(h_{\varepsilon}^{+}-h\right) d x=\varepsilon \int_{A_{k}}\left(h_{\varepsilon}^{+}-h-k\right) d x .
$$

If $1<p<n$, using monotonicity and Sobolev as above, this yields

$$
\left(\int_{A_{k}}\left|h_{\varepsilon}^{+}-h-k\right|^{\frac{p n}{n-p}} d x\right)^{(n-p) / n} \leq C \varepsilon \int_{A_{k}}\left(h_{\varepsilon}^{+}-h-k\right) d x
$$

with some constant $C=C(p, n, \Omega)$. Hence, by Hölder, we have

$$
\begin{aligned}
\int_{A_{k}}\left(h_{\varepsilon}^{+}-h-k\right) d x & \leq\left|A_{k}\right|^{1-\frac{n-p}{p n}}\left(\int_{A_{k}}\left|h_{\varepsilon}^{+}-h-k\right|^{\frac{p n}{n-p}} d x\right)^{\frac{n-p}{p n}} \\
& \leq C \varepsilon^{1 / p}\left|A_{k}\right|^{1-\frac{n-p}{p n}}\left(\int_{A_{k}}\left(h_{\varepsilon}^{+}-h-k\right) d x\right)^{1 / p},
\end{aligned}
$$

or, equivalently,

$$
\int_{A_{k}}\left(h_{\varepsilon}^{+}-h-k\right) d x \leq C \varepsilon^{1 /(p-1)}\left|A_{k}\right|^{1+\frac{p}{n(p-1)}} .
$$

If $p=n$, we use the inequality

$$
\int_{A_{k}} \exp \left(\frac{\left|\left(h_{\varepsilon}^{+}-h-k\right)\right|}{C\left\|D\left(h_{\varepsilon}^{+}-h\right)\right\|_{n}}\right)^{n /(n-1)} d x \leq C\left|A_{k}\right| .
$$

Combined with Jensen's inequality and the energy estimate above it gives

$$
\int_{A_{k}}\left|\left(h_{\varepsilon}^{+}-h-k\right)\right|^{n /(n-1)} d x \leq C \varepsilon^{1 /(n-1)}\left\|h_{\varepsilon}^{+}-h-k\right\|_{1, A_{k}}^{1 /(n-1)}\left|A_{k}\right|,
$$

which in turn implies by Hölder that

$$
\int_{A_{k}}\left(h_{\varepsilon}^{+}-h-k\right) d x \leq C \varepsilon^{1 /(n-1)}\left|A_{k}\right|^{n /(n-1)} .
$$

This is (4.5) for $p=n$. 
Let us denote

$$
f(k)=\int_{A_{k}}\left(h_{\varepsilon}^{+}-h-k\right) d x=\int_{k}^{\infty}\left|A_{t}\right| d t,
$$

whence

$$
f^{\prime}(k)=-\left|A_{k}\right|
$$

for almost every $k$. Thus (4.5) can be rewritten as

$$
f(k) \leq C \varepsilon^{1 /(p-1)}\left(-f^{\prime}(k)\right)^{1+\frac{p}{n(p-1)}} .
$$

If $f(k) \neq 0$ on $\left[k_{1}, k_{2}\right]$, this yields

$$
1 \leq C \varepsilon^{\frac{n}{n(p-1)+p}} \frac{-f^{\prime}(k)}{f(k)^{\frac{n(p-1)}{n(p-1)+p}}} .
$$

Hence

$$
k_{2}-k_{1} \leq C \varepsilon^{\frac{n}{n(p-1)+p}}\left[f\left(k_{1}\right)^{\frac{p}{n(p-1)+p}}-f\left(k_{2}\right)^{\frac{p}{n(p-1)+p}}\right] .
$$

In particular, if we choose $k_{1}=0$, then as

$$
f(0)=\int_{A_{0}} h_{\varepsilon}^{+}-h d x=\int_{\Omega}\left|h_{\varepsilon}^{+}-h\right| d x
$$

by the comparison principle, (4.7) gives

$$
k_{2} \leq C \varepsilon^{\frac{n}{n(p-1)+p}}\left(\int_{\Omega}\left|h_{\varepsilon}^{+}-h\right| d x\right)^{\frac{p}{n(p-1)+p}}
$$

for all $k_{2}>0$ for which $f\left(k_{2}\right)>0$. Thus we obtain

$$
\left\|h_{\varepsilon}^{+}-h\right\|_{\infty, \Omega} \leq C \varepsilon^{\frac{n}{n(p-1)+p}}\left\|h_{\varepsilon}^{+}-h\right\|_{1, \Omega}^{\frac{p}{n(p-1)+p}} .
$$

This together with (4.4) finally yields

$$
\left\|h_{\varepsilon}^{+}-h\right\|_{\infty, \Omega} \leq C \varepsilon^{\frac{n}{n(p-1)+p}} \varepsilon^{\frac{1}{p-1} \frac{p}{n(p-1)+p}}=C \varepsilon^{1 /(p-1)}
$$

as claimed.

Remark 4.2. If $p<2$, then the above argument has to be modified slightly. Using the fact that

$$
(p-1) \frac{|a-b|^{2}}{(|a|+|b|)^{2-p}} \leq\left(|a|^{p-2} a-|b|^{p-2} b\right) \cdot(a-b),
$$

which is valid for $1<p<2$, we obtain

$$
\int_{\Omega} \frac{\left|D\left(h_{\varepsilon}^{+}-h\right)\right|^{2}}{\left(\left|D h_{\varepsilon}^{+}\right|+|D h|\right)^{2-p}} d x \leq C \varepsilon \int_{\Omega}\left|h_{\varepsilon}^{+}-h-k\right| d x .
$$

By Hölder, this implies

$$
\left(\int_{\Omega}\left|D\left(h_{\varepsilon}^{+}-h\right)\right|^{p} d x\right)^{2 / p} \leq C \varepsilon \int_{\Omega}\left|h_{\varepsilon}^{+}-h-k\right| d x,
$$

where the constant $C$ depends also on the energies $\left\|D h_{\varepsilon}^{+}\right\|_{p, \Omega}$ and $\|D h\|_{p, \Omega}$. Arguing now as above, we arrive (instead of (4.5)) to

$$
\int_{A_{k}}\left(h_{\varepsilon}^{+}-h-k\right) d x \leq C \varepsilon\left|A_{k}\right|^{2\left(1-\frac{n-p}{n p}\right)} .
$$


If $2>p>\frac{2 n}{2 n-1}$, then we can write $2\left(1-\frac{n-p}{n p}\right)=1+\alpha$ for some $\alpha>0$, and in terms of the function $f(k)=\int_{A_{k}}\left(h_{\varepsilon}^{+}-h-k\right) d x(4.9)$ takes the form

$$
f(k) \leq C \varepsilon\left(-f^{\prime}(k)\right)^{1+\alpha} \quad \text { for a.e. } k \text {. }
$$

Upon integration, this leads to the estimate

$$
\left\|h_{\varepsilon}^{+}-h\right\|_{\infty, \Omega} \leq C \varepsilon^{\frac{1}{1+\alpha}}\left\|h_{\varepsilon}^{+}-h\right\|_{1, \Omega}^{\frac{\alpha}{1+\alpha}} .
$$

Since (4.8) and Sobolev's inequality imply that $\left\|h_{\varepsilon}^{+}-h\right\|_{1, \Omega} \leq C \varepsilon$, we finally have that

$$
\left\|h_{\varepsilon}^{+}-h\right\|_{\infty, \Omega} \leq C \varepsilon,
$$

provided that $2>p>\frac{2 n}{2 n-1}$. Moreover, unlike in the case $p>2$, the constant $C$ depends on the energies $\left\|D h_{\varepsilon}^{+}\right\|_{p, \Omega}$ and $\|D h\|_{p, \Omega}$.

\section{REFERENCES}

[1] G. Akagi, P. Juutinen, And R. KajikiYA, Asymptotic behavior of viscosity solutions for a degenerate parabolic equation associated with the infinity-Laplacian, Math. Ann. 343 (2009), no. 4, 921-953.

[2] A. Banerjee, and N. Garofalo, Gradient bounds and monotonicity of the energy for some nonlinear singular diffusion equations, arXiv:1202.5068.

[3] Berestycki, H., Nirenberg, L., Varadhan, S. R. S., The principal eigenvalue and maximum principle for second-order elliptic operators in general domains, Comm. Pure Appl. Math. 47 (1994), no. 1, 47-92.

[4] Birindelli, I., And Demengel, F., Eigenvalue, maximum principle and regularity for fully nonlinear homogeneous operators, Commun. Pure Appl. Anal. 6 (2007), no. 2, 335-366.

[5] Y. Chen, Y. Giga, And S. Goto, Uniqueness and existence of viscosity solutions of generalized mean curvature flow equations, J. Differential Geom., 33 (1991), pp. 749-786.

[6] K. Does, An evolution equation involving the normalized $p$-Laplacian, Commun. Pure Appl. Anal. 10 (2011), no. 1, 361-396.

[7] L. C. Evans, And J. Spruck, Motion of level sets by mean curvature. I., J. Differential Geom. 33 (1991), no. 3, 635-681.

[8] Giga, Y., Surface Evolution Equations. A Level Set Approach. Monographs in Mathematics, 99. Birkhäuser Verlag, Basel, 2006.

[9] O. Ladyzhenskaja, And N. URAL'tseva, Linear and quasilinear elliptic equations, Academic Press, New York, 1968.

[10] G. M. LiEBERMAn, Boundary regularity for solutions of degenerate elliptic equations, Nonlinear Anal., 12 (1988), pp. 1202-1219.

[11] P. Lindqvist, On a nonlinear eigenvalue problem. Fall School in Analysis (Jyväskylä, 1994), 33-54, Report, 68, Univ. Jyväskylä, Jyväskylä, 1995.

[12] J.J. Manfredi, M. Parviainen, And J. D. Rossi, An asymptotic mean value characterization for a class of nonlinear parabolic equations related to tug-of-war games, SIAM J. Math. Anal. 42 (2010), no. 5, 2058-2081.

Department of Mathematics and Statistics, P.O.Box 35, Fin-40014 UniverSITY OF JYVÄSKYLÄ, FINLAND

E-mail address: petri.juutinen@jyu.fi 